\title{
Laboratory-based Edge-illumination Phase-contrast Imaging: Dark-field Retrieval and High-resolution Implementations
}

\author{
Marco Endrizzi, Paul C. Diemoz, Charlotte K. Hagen, Thomas P. Millard, Fabio A. Vittoria, Ulrich H. Wagner, \\ Christoph Rau, Ian K. Robinson and Alessandro Olivo
}

\begin{abstract}
Edge illumination is an X-ray phase-contrast imaging technique capable of quantitative retrieval of phase and amplitude images. The retrieval of the ultra-small-angle $X$-ray scattering was recently developed and implemented with the area-imaging counterpart of an edge-illumination system, sometimes referred to as coded-aperture. This is an incoherent and achromatic technique, well suited for translation of the potential of X-ray phase contrast imaging into efficient laboratory-scale setups. We report on recent advances of these developments along two main directions. One relates to the expansion of the technique with respect to the data analysis and corrections that are required when non-ideal optical elements are used and optimized sampling strategies. The second is directed towards high-resolution and high-energy implementations. A laboratory-based prototype for high-energy X-ray phase-contrast microscopy was built and its performance was modelled and experimentally characterized.
\end{abstract}

\section{INTRODUCTION}

$X^{-19}$ -RAY phase contrast imaging (XPCI) has the potential to transform applications of X-ray imaging in many fields, including medicine, biology, material sciences and security inspections. Following pioneering studies with synchrotron and microfocus X-ray sources [1-8], methods have emerged which enable the implementation of XPCI with conventional laboratory sources $[9,10]$. We report here on the recent developments of edge illumination [7] and its laboratory-based counterpart, sometimes also called coded-aperture [10] XPCI. Edge illumination and coded-aperture are XPCI techniques capable of quantitative retrieval of absorption, phase [11], and ultra-small-angle X-ray scattering [12]. They were shown to be incoherent and achromatic, enabling an efficient

Manuscript received November 14, 2014. This work was supported by the United Kingdom Engineering and Physical Sciences Research Council (Grant Numbers EP/I022562/1 and EP/I021884/1). M. Endrizzi and P. C. Diemoz are supported by Marie Curie Career Integration Grants within the Seventh Framework of the European Union , Grant Numbers PCIG12-GA2012-333990 and PCIG12-GA-2012-334056.

M. Endrizzi, P. C. Diemoz, C. K. Hagen, T. P. Millard, F. A. Vittoria are with the Department of Medical Physics and Biomedical Engineering, University College London, Malet Place, Gower Street, London WC1E 6BT, United Kingdom (e-mail:m.endrizzi@ucl.ac.uk).

Ulrich H. Wagner, Christoph Rau are with the Diamond Light Source, Harwell Oxford Campus, OX11 0DE Didcot, United Kingdom

I. K. Robinson is with the London Centre for Nanotechnology, WC1H 0AH London, United Kingdom and the Research Complex at Harwell, Harwell Oxford Campus, OX11 0FA Didcot, United Kingdom implementation also by using standard rotating anode X-ray tube sources [13-15]. They require two absorbing masks, with relatively large pitches, matched with the detector such that a one-to-one relationship exists between each of the apertures in the masks and columns of the detector pixels. A laboratorybased system built in this way provides high sensitivity, it is efficient with respect to the dose delivered to sample and it robust against environmental vibrations and thermal stress [16-19]. The method was recently extended from planar to three-dimensional imaging, using both synchrotron radiation and laboratory-based X-ray sources [20-22]

\section{MATERIALS AND METHODS}

The typical experimental set-up is depicted in Fig. 1. An Xray beam, generated by a rotating anode tube, is shaped by the pre-sample mask, traverses a sample and it is analyzed by the detector mask before being recorded by the arrays of pixels of a digital detector.
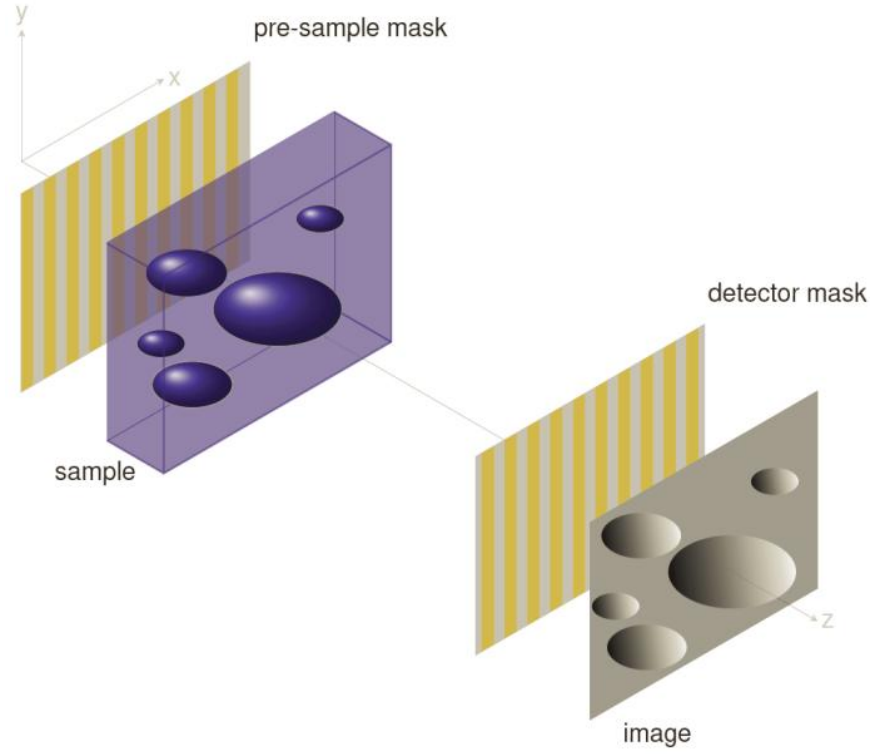

Fig. 1. Sketch of the experimental setup. An X-ray beam generated by a rotating anode X-ray tube is shaped into an array of independent laminar beams by an absorbing mask that is positioned before the sample. This array of beams is then analyzed by a second mask before being recorded by a digital detector. 
The masks are composed of absorbing material, typically gold, with apertures that allow for the transmission of X-rays, effectively shaping the beam into an array of laminar beams. The substrate used in the fabrication of the masks is usually graphite, in order to maximize the transmission through both the pre-sample and the detector masks. The masks are aligned such that each of these laminar beams impinges on the edge of the apertures in the detector mask.

Absorption, refraction and ultra-small-angle X-ray scattering in the sample are measured by tracking the changes in the shape and position of each of these laminar beams. When an extended source is used, the image formation can be described by using geometrical optics and considering each aperture independent from each other [12]:

$$
\frac{I(x)}{I_{0}}=t(O * L)\left(x-\Delta x_{R}\right)
$$

where $I_{0}$ is the intensity that passes through the pre-sample aperture, $t$ is the fraction of intensity transmitted by the object and $O(x)$ is the function describing the scattering introduced by the sample. When refraction occurs, the detector aperture appears shifted by an amount $\Delta x_{R}=-\Delta \theta_{R} z_{o d}$ with respect to the beam $\left(z_{o d}\right.$ is the distance between the object and the detector and $\Delta \theta_{R}$ is the angle of deflection): this is due to the deflection of the beam locally caused by the refraction in the sample. The illumination function $L(x)$ describes the change in the detected beam intensity as a function of the displacement $x$ between pre-sample and detector mask. By acquiring three images by setting the masks to different offsets one with respect to the other $\left(x_{2}=0\right.$ and $\left.x_{1}=-x_{3}\right)$, the system of equations [12]:

$$
I_{i}=t \frac{A_{M N}}{\sqrt{2 \pi \sigma_{M N}^{2}}} \exp \left[-\frac{\left(x-\Delta x_{R}\right)^{2}}{2 \sigma_{M N}^{2}}\right] ; i=1,2,3
$$

can be analytically solved for $t, \Delta x_{R}$ and $\sigma_{M N}^{2}$ when a singleGaussian approximation is chosen for the illumination function $L(x)=A_{N}\left(1 / \sqrt{2 \pi \sigma_{N}^{2}}\right) \exp \left(-x^{2} / 2 \sigma_{N}^{2}\right)$ and for the scattering function $O(x)=A_{M}\left(1 / \sqrt{2 \pi \sigma_{M}^{2}}\right) \exp \left(-x^{2} / 2 \sigma_{M}^{2}\right)$. One problem that was encountered is that, if the illumination function does not reach zero at the tails, the retrieval cannot correctly separate the contributions to the projection images coming from absorption, refraction and scattering. A singleGaussian term reaches zeros on the tails of the distribution thus, if an offset is present in the illumination function, there is an excess of intensity that it is not accounted for by using Eq. (2). This can be taken into account by modifying Eq. (2) in the following way [23]:

$$
I_{i}=t \frac{A_{M N}}{\sqrt{2 \pi \sigma_{M N}^{2}}} \exp \left[-\frac{\left(x-\Delta x_{R}\right)^{2}}{2 \sigma_{M N}^{2}}\right]+t c
$$

where $c$ indicates the offset of the illumination function, which is assumed to be known, at least by experimental measurement. This system can be solved by iteratively correcting for the quantity $t c$ directly in the projection images. The transmission of the sample $t$ is usually unknown but it can be calculated the first time using the solution of Eq. (2) and then iteratively refined by using the set of equations [23]:

$$
\begin{gathered}
t^{k+1}=\frac{2 x_{1}}{A_{M N}} \sqrt{\frac{\pi}{D^{k}+C^{k}}} I_{2} \exp \left[\frac{1}{2^{4}} \frac{\left(D^{k}+C^{k}\right)^{2}}{D^{k}+C^{k}}\right] \\
\Delta x_{R}^{k+1}=\frac{x_{1}}{2} \frac{D^{k}-C^{k}}{D^{k}+C^{k}} \\
{\sigma_{M}^{2}}^{k+1}=\frac{2 x_{1}^{2}}{D^{k}+C^{k}}-\sigma_{N}^{2}
\end{gathered}
$$

where the substitutions $\left.C^{k}=-2 \ln \left[\left(I_{1}-I_{r}\right) /\left(I_{2}-I_{r}\right)\right]\right)$, $\left.D^{k}=-2 \ln \left[\left(I_{3}-I_{r}\right) /\left(I_{2}-I_{r}\right)\right]\right)$ and $I_{r}=t^{k} c\left[1-\left(1 / 2^{k}\right)\right]$ were used.

In order to push the resolution limit of few tens of micrometers, a prototype for high-resolution XPCI was designed, modelled and tested in our laboratory. It is based on a strongly demagnified (10x) pre-sample mask and uses a microfocal X-ray source [24]. The resolution was measured by means of a star-pattern phase-object and compared with a wave optics simulation of the whole imaging system. The accuracy of the retrieval was tested by using a two-material sample of known shape and composition.

\section{RESULTS}

The minimum number of projection images required for retrieving absorption, refraction and ultra-small-angle scattering is three, acquired by using different displacements $x_{1,3}$ between the pre-sample and the detector mask. The optimization of the acquisition of these projection images was studied by means of a numerical simulation. An ideal singlephoton counting detector was considered and the noise implemented as Poisson statistics fluctuations. The total number of photons allowed through the pre-sample aperture was kept constant while changing $x_{1}$ and $x_{3}$. This provides information for the optimization of the data acquisition in our prototype, and could be adapted for the design or optimization of any edge-illumination set-up. The results of this series of simulations are shown in Fig. 2, the position of the minimum identifies the settings that yield the highest signal-to-noise ratio for a given photon flux. The geometry used in the simulation was $1.6 \mathrm{~m}$ from the source to the pre-sample mask, $2 \mathrm{~m}$ source to detector distance, 70 micrometers source size, 12 and 67 micrometers for the pre-sample mask's aperture and pitch and 20 and 84 micrometers for the detector mask's aperture and pitch. 

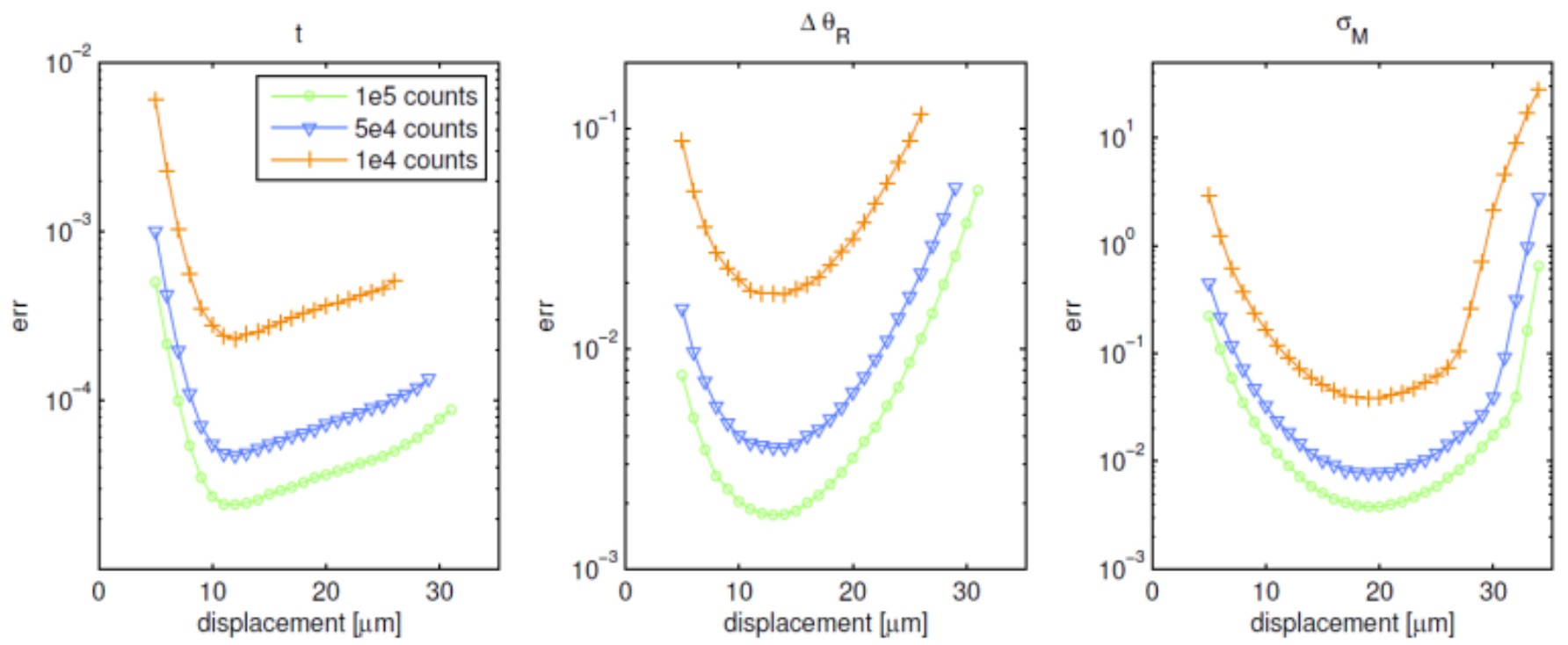

Fig. 2. Numerical simulation of the noise propagation in the retrieved images. The photon statistics is kept constant while changing the displacement between the sample and detector mask.

The retrieval was expanded to include non-ideal sources and optical elements. When this occurs an offset could be present in the illumination function and can be taken into account by using the set of Eqs. (3)-(6). This process was simulated for a known numerical phantom and the error between the retrieved images and the known solution was calculated after iteration. The results are shown in Fig. 3. The error between the known numerical sample $S_{i j}$ and the retrieved one $R_{i j}$ is calculated as err $=(1 / N) \sum_{i j}\left(S_{i j}-R_{i j}\right)^{2}$ and it is plotted as function of the number of iterations $k$. The indices $i$ and $j$ cover the pixels of the image and $N$ is their total number

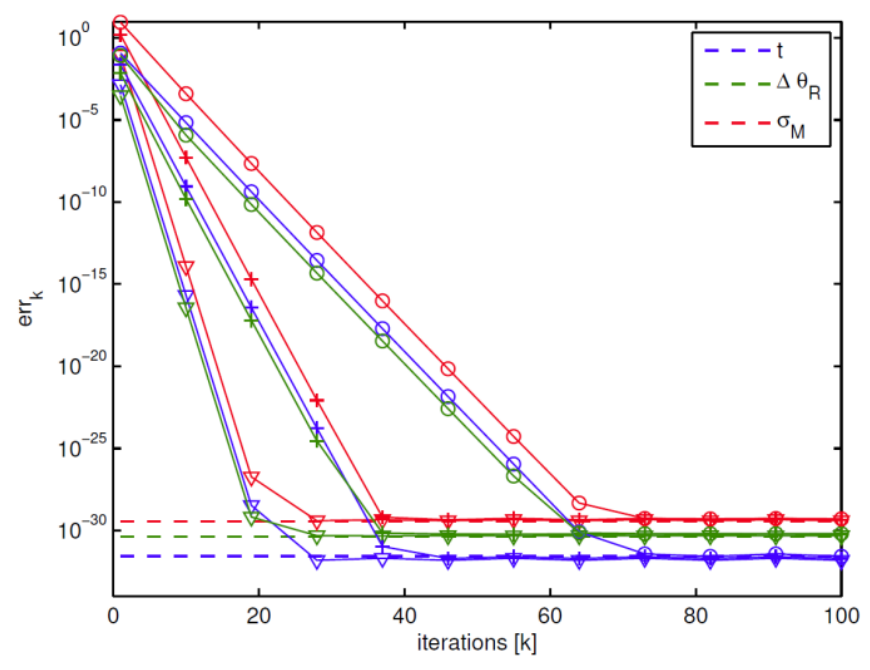

Fig. 3. Convergence of the iterative solution for $10 \%$ (triangles), $20 \%$ (crosses) and 30\% (circles) offset in the illumination curve.

The retrieved values (solid lines) converge to the exact solution (dashed lines) within a few tens of iterations. Blue,

green and red respectively refer to absorption, refraction and scattering; triangles, crosses and squares refer to $10 \%, 20 \%$ and $30 \%$ offset on the tails, respectively. This shows the robustness of the imaging method and of the developed retrieval algorithm.

With the high-resolution setup [24], a 1.5 um minimum separation between dark and bright fringes was measured by using a phase star pattern. This was confirmed by a polychromatic wave optics simulation [25], which showed good agreement with the experimental data. These results were achieved at the high X-ray energy of $80 \mathrm{kVp}$ on a tungsten target, at which the origin of the signal can be considered to be pure phase contrast. An example of refraction image of two boron fibers crossing each other is shown below, in Fig. 4.

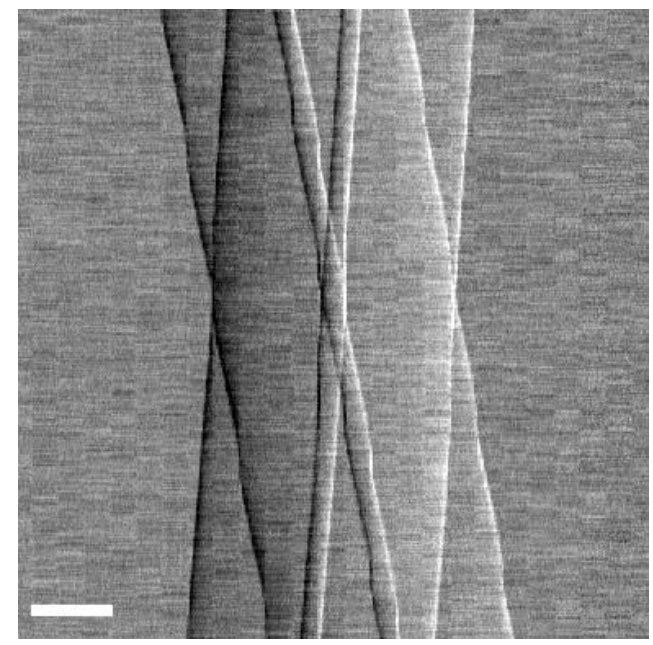

Fig. 4. Refraction image of two boron fibers (with tungsten core) crossing each other. The image was acquired at $80 \mathrm{kVp}$, the scale bar is 50 micrometers. 


\section{CONCLUSIONS}

Edge-illumination and coded-aperture are noninterferometric and achromatic X-ray phase-contrast imaging techniques, capable of simultaneously retrieving absorption, differential-phase and ultra-small-angle X-ray scattering. The optimization of the data acquisition scheme was studied based on numerical simulation results. The retrieval algorithm was expanded to account for the offset that is present in the illumination function when the masks allow a fraction of the radiation to go through the absorbing septa. A high-resolution set-up provided micrometer spatial resolution with X-ray energies up to $80 \mathrm{keV}$.

\section{REFERENCES}

[1] U. Bonse and M. Hart, "An X-ray interferometer", Applied Physics Letters 6, pp. 155-156, 1965

[2] A. Snigirev, I. Snigireva, V. Kohn, S. Kuznetsov, and I. Schelokov, "On the possibilities of X-ray phase contrast microimaging by coherent highenergy synchrotron radiation", Review of Scientific Instruments 66, pp. 5486-5492, 1995.

[3] V.N. Ingal and A. Beliaevskaya, "X-ray phase-wave topography observation of the phase contrast from a non-crystalline object," Journal of Physics D: Applied Physics 28, pp. 2314-2317, 1995.

[4] A. Momose, T. Takeda, Y. Itai, and K. Hirano, "Phase-contrast X-ray computed tomography for observing biological soft tissues", Nature Medicine 2, pp. 473-475, 1996.

[5] S. W. Wilkins, T. E. Gureyev, D. Gao, A. Pogany, and A. W. Stevenson, "Phase-contrast imaging using polychromatic hard X-rays", Nature 384, pp. 335-338, 1996.

[6] T. Davis, D. Gao, T. E. Gureyev, A. W. Stevenson, and S. W. Wilkins, "Phase-contrast imaging of weakly absorbing materials using hard Xrays", Nature, vol. 373, issue 6515, pp. 595-598, 1995.

[7] A. Olivo, F. Arfelli, G. Cantatore, R. Longo, R. H. Menk, S. Pani, M. Prest, P. Poropat, L. Rigon, G. Tromba, E. Vallazza, and E. Castelli, “An innovative digital imaging set-up allowing a low-dose approach to phase contrast applications in the medical field", Medical Physics 28, pp. 1610-1619, 2001

[8] C. David, B. Nohammer, H. H. Solak, and E. Ziegler, "Differential Xray phase contrast imaging using a shearing interferometer", Applied Physics Letters 81, pp. 3287-3289, 2002.

[9] F. Pfeiffer, T. Weitkamp, O. Bunk, and C. David, "Phase retrieval and differential phase-contrast imaging with low-brilliance X-ray sources", Nature Physics 2, pp. 258-261, 2006.

[10] A. Olivo and R. D. Speller, "A coded-aperture technique allowing X-ray phase contrast imaging with conventional sources", Applied Physics Letters, 91, pp. 074106, 2007.

[11] P. R. T. Munro, K. Ignatyev, R. D. Speller, and A. Olivo, "Phase and absorption retrieval using incoherent X-ray sources", Proc. Natl. Acad. Sci. USA, 109, pp. 13922, 2012.

[12] M. Endrizzi, P. C. Diemoz, T. P. Millard, J. Louise Jones, R. D. Speller, I. K. Robinson and A. Olivo, "Hard X-ray dark-field imaging with incoherent sample illumination", Applied Physics Letters, 104, pp. 024106, 2014.

[13] M. Endrizzi, P. Diemoz, C. Hagen, F. Vittoria, P. Munro, L. Rigon, D. Dreossi, F. Arfelli, F. Lopez, R. Longo, M. Marenzana, P. Delogu, A. Vincenzi, L. D. Ruvo, G. Spandre, A. Brez, R. Bellazzini, and A. Olivo, "Edge-illumination X-ray phase contrast imaging: matching the imaging method to the detector technology". Journal of Instrumentation, 9(11), pp. C11004, 2014.

[14] M. Endrizzi, P. Diemoz, M. Szafraniec, C. Hagen, T. Millard, C. Zapata, P. Munro, K. Ignatyev, M. Marenzana, R. Speller, et al. Edge illumination and coded-aperture X-ray phase-contrast imaging: increased sensitivity at synchrotrons and lab-based translations into medicine, biology and materials science. In SPIE Medical Imaging, pp. 866812-866812, 2013.

[15] M. Endrizzi, P. Diemoz, P. Munro, C. Hagen, M. Szafraniec, T. Millard, C. Zapata, R. Speller, and A. Olivo. Applications of a noninterferometric X-ray phase contrast imaging method with both synchrotron and conventional sources. Journal of Instrumentation, 8(05), pp. C05008, 2013.

[16] M. Marenzana, C. K. Hagen, P. D. N. Borges, M. Endrizzi, M. B. Szafraniec, K. Ignatyev and A. Olivo, "Visualization of small lesions in rat cartilage by means of laboratory-based X-ray phase contrast imaging", Physics in Medicine and Biology, 57, 24, pp. 8173-8184, 2012.

[17] A. Olivo, S. Gkoumas, M. Endrizzi, C.K. Hagen, M.B. Szafraniec, P.C. Diemoz, P.R.T. Munro, K. Ignatyev, B. Johnson, J.A. Horrocks, S.J. Vinnicombe, J.L. Jones and R.D. Speller, "Low-dose phase contrast mammography with conventional sources," Medical Physics 40(9), pp. 090701, 2013.

[18] P. C. Diemoz, C. K. Hagen, M. Endrizzi, and A. Olivo, "Sensitivity of laboratory based implementations of edge illumination X-ray phasecontrast imaging", Applied Physics Letters, 103, pp. 244104, 2013.

[19] T.P. Millard, M. Endrizzi, K. Ignatyev, C.K. Hagen, P.R.T. Munro, R.D. Speller and A. Olivo, "Method for automatization of the alignment of a laboratory based X-ray phase contrast edge illumination system," Review of Scientific Instruments 84, pp. 083702, 2013.

[20] C.K. Hagen, P.R.T. Munro, M. Endrizzi, P.C. Diemoz and A. Olivo, "Low-dose phase contrast tomography with conventional sources", Medical Physics 41(7), pp.070701 (2014).

[21] C.K. Hagen, P.C. Diemoz, M. Endrizzi, L. Rigon, D. Droessi, F. Arfelli, F.C.M. Lopez, R. Longo and A. Olivo, "Theory and preliminary experimental verification of quantitative edge illumination X-ray phase contrast tomography", Optics Express 22(7), pp. 7989-8000, 2014.

[22] C.K. Hagen, P.C. Diemoz, M. Endrizzi and A. Olivo, "The effect of the spatial sampling rate on quantitative phase information extracted from planar and tomographic edge illumination X-ray phase contrast images," Journal of Physics D: Applied Physics 47, pp. 455401, 2014.

[23] M. Endrizzi and A. Olivo, "Absorption, refraction and scattering retrieval with an edge-illumination-based imaging setup". Journal of Physics D: Applied Physics, accepted for publication.

[24] M. Endrizzi, F. A. Vittoria, P. C. Diemoz, R. Lorenzo, R. D. Speller, U. H. Wagner, C. Rau, I. K. Robinson, and A. Olivo, "Phase-contrast microscopy at high X-ray energy with a laboratory setup". Optics Letters, 39(11) pp. 3332-3335, 2014.

[25] F. A. Vittoria, P. C. Diemoz, M. Endrizzi, R. Luigi, F. C. Lopez, D. Dreossi, P. R. T. Munro and A. Olivo, "Strategies for efficient and fast wave optics simulation of coded-Aperture and other X-ray phasecontrast imaging methods", Applied Optics 52, 28, pp. 6940-6947, 2013. 